\title{
Abriendo Caminos para Colombia: La función SOCIAL DE SATENA EN UN ESCENARIO DE POSCONFLICTO
}

\author{
ADRIANA LUCÍA MARÍN RAMÍREZ \\ DANIEL GARZÓN MORENO ${ }^{2}$ \\ CAMILO ANDRÉS CASTIBLANCO ${ }^{3}$
}

\section{INTRODUCCIÓN}

La responsabilidad y función social de una aerolínea como Satena va más allá de cumplir satisfactoriamente con el traslado de los pasajeros de un destino a otro. Satena lleva cumpliendo por más de cincuenta años una labor social de abrir caminos para el desarrollo de las zonas periféricas, menos favorecidas y más afectadas por el conflicto armado de Colombia. Satena ha sufrido las consecuencias de la crisi económica, de la competencia comercial y del alza del dólar, no por eso ha dejado de buscar la manera de poder comunicar el centro del país con los territorios más alejados del país, para dar apoyo a la población más necesitada.

Este artículo busca realizar un breve recorrido histórico de la aerolínea enmarcando y enfatizando la función social que ha desempeñado en Colombia a lo largo de su trayectoria. De igual manera, se proyecta hacer una descripción social de la población que habita en las tres regiones donde Satena tiene mayor presencia y cobertura. Las regiones Pacífico, Orinoquia y Amazónica están caracterizadas por ser habitadas por la mayoría de la población étnica de Colombia y por tener los porcentajes más altos de las Necesidades Básicas Insatisfechas, de ahí que el apoyo otorgado por la aerolínea ha sido fundamental durante el conflicto armado y se esté preparando para un escenario de posconflicto y los desafíos que esto conlleva. 
Para el desarrollo de los temas mencionados, el grupo de investigación de la Dirección de Análisis de Contexto y Posconflicto (DICOP) de la Fuerza Aérea le realizó una entrevista al Mayor General de la Fuerza Aérea Pedro Ignacio Lozano Quinche, actual presidente de la aerolínea Satena, quien explicó en breves pero contundentes palabras cuál ha sido la función social de la aerolínea desde su nacimiento en 1962, y la importancia que ha tenido para el desarrollo de las regiones más alejadas del centro del país. Desde el apoyo técnico de la Fuerza Aérea Colombiana con la disposición de aviones, pilotos, técnicos mecánicos y administrativos; y el respaldo financiero del gobierno nacional, Satena surgió con el único propósito de brindar apoyo y unir a los lugares más alejados y periféricos de Colombia con las principales ciudades del centro del país. Dicho propósito ha sido el eje por el cual Satena se ha mantenido hasta la actualidad, no sin antes haber pasado por graves crisis económicas por causa de los cambios de las políticas del gobierno en el acompañamiento financiero a la empresa que tuvo inicio en 1991 al establecer que Satena debería ser una entidad autónoma.

Dicha decisión afectó en diferentes sentidos a la aerolínea, tal vez el más grave de las afectaciones según el Mayor General Lozano, fue el descuido y abandono en el que se vieron inmersas las ciudades periféricas a las que solo llega Satena por tener que cancelar rutas que unían los municipios y veredas con las capitales de los departamentos más alejados, destinos que fueron apropiados por los vuelos chárter incrementando así el valor de cada pasaje lo que impide a los habitantes de dichas zonas con menos recursos poder viajar por vía aérea. Debido a esto, a Satena le tocó empezar a competir por las rutas más concurridas con las demás aerolíneas que por su naturaleza comercial, son más grandes y fuertes económicamente. Esta decisión del gobierno, que para el Mayor General Lozano fue un grave "error", le costó también a Satena por más de quince años un endeudamiento que lo tuvo al borde de la quiebra en el 2010.

Después de llegar a tan críticos momentos financieros, y de mantener viva la aerolínea estable pero sin muchas ganancias, el Banco Interamericano de Desarrollo (BID) realizó un estudio en el año 2015, en el que se estableció que para el debido funcionamiento de la aerolínea y para asegurar el cumplimiento de su función social con el país, el gobierno debía volver a apoyar y a ayudar a Satena otorgándole una subvención para poderse liberar de deudas y apoyar la gestión para cubrir nuevas rutas y llegar cada vez a más destinos. El Mayor General Lozano sabe que con el venir del tiempo y con la nueva ayuda del gobierno, la cobertura a nivel nacional iba a ser mayor y así se podría mantener, recuperar viejas rutas que unieran los pequeños municipios con las capitales de los departamentos, y así, incrementar el impacto social de Satena como una empresa indispensable para el país. 


\section{"Hay muchos lugares de la geografía colombiana que necesitan de nuestro apoyo"}

En la actualidad Satena cubre 34 destinos a nivel nacional (Ver Mapa) de los cuales diez hacen parte de la región Amazónica, ocho de la región Pacífico, seis de la Orinoquia, seis de la Andina y cuatro de la región Caribe. De esos destinos, la mayoría se encuentra en zonas geográficas de difícil acceso en Colombia por lo que la misión social de la aerolínea se enmarca en conectar y apoyar la población que vive en territorios fronterizos y periféricos con las principales ciudades del país, entre ellas Villavicencio, Bucaramanga, Cali, Medellín y Bogotá. En las tres regiones donde Satena tiene mayor cobertura, (Pacífico, Amazónica y Orinoquia), son precisamente las que tienen un mayor índice de población étnica como la indígena y la afrodescendiente. Al mismo tiempo, son regiones en donde el porcentaje de las Necesidades Básicas Insatisfechas (NBI) son muy altas como se va a describir a continuación.

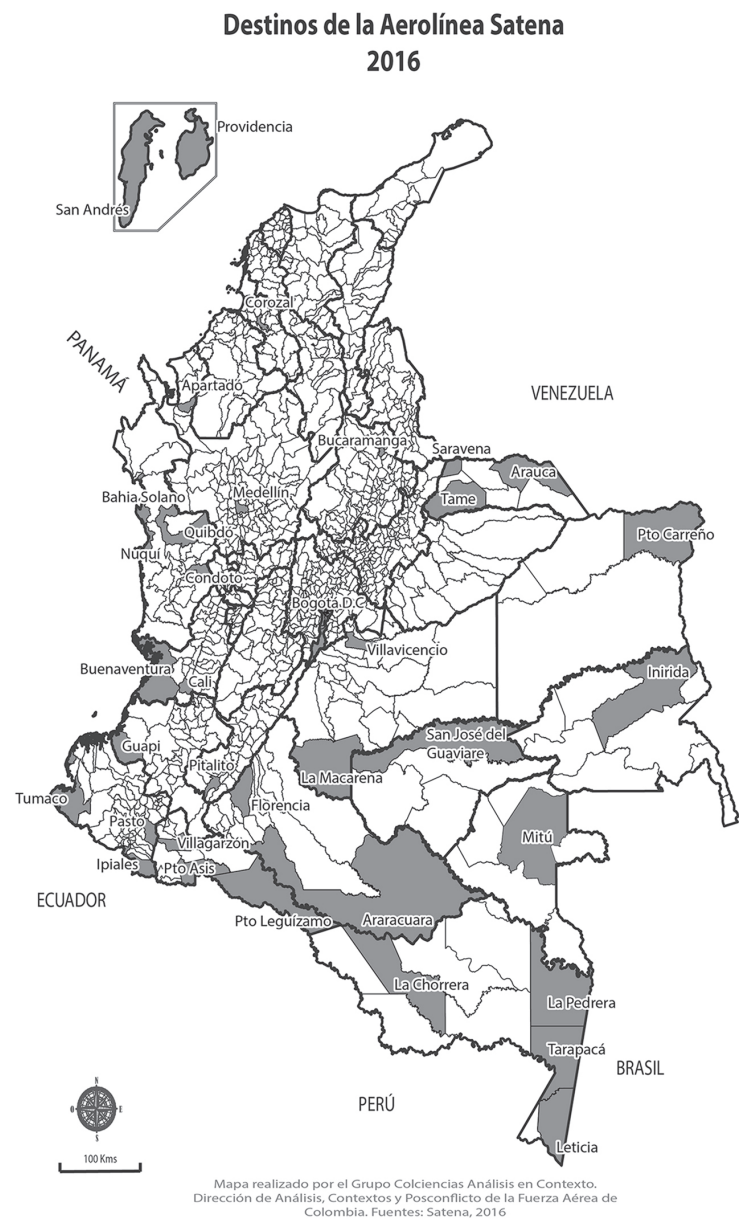




\section{- Región Pacífico}

La región Pacífico es una región de contrastes, en donde claramente los cuatro departamentos que la integran se encuentran en niveles distintos en lo referente a pobreza (DANE, 2016). Los niveles más críticos se encuentran en el departamento de Choco (79,19\%), en donde las NBI son marcadamente críticas, teniendo como vecino a un departamento como el Valle del Cauca (15,68\%), que se encuentra en la franja de desempeño más alto, en el marco nacional. Por el otro lado, departamentos como Cauca (46,62\%) y Nariño (43,79\%) se encuentran en un rango medio alto, constituyéndose bajo el promedio de muchas de las regiones colombianas. Frente a la población étnica, la presencia de comunidades indígenas está repartida en distintos lugares del territorio. Se puede señalar que existe una fuerte presencia en las zonas norte, desde la frontera panameña hasta el Valle del Cauca. En esta zona se encuentran comunidades como Embera Dodiba, Embera Chamí y Waunaan. Un segundo grupo se puede encontrar en el suroriente, en el Cauca, en donde se ubican comunidades como los Tototoe, Guambianos, Guanaca, Kokonukos y Yanaconá, y en el sur de la región, en el departamento de Nariño, se encuentra la comunidad Awa.

Del mismo modo, la región Pacífico es una de las cuales cuenta con la mayor presencia de comunidades afrocolombianas la cual cubre desde el norte hasta el sur del departamento, en la línea costera; en la zona montañosa de estos departamentos su presencia baja significativamente. En esta región existe la mayor concentración de las comunidades afrocolombianas en Colombia. Estos territorios se encuentran, entre los departamentos de Chocó, Valle del Cauca, Cauca y Nariño, y se ubican en la franja occidental.

Por otro lado, el Pacífico colombiano reporta una situación más grave y preocupante en términos de víctimas, configurándose como una de las regiones más violentas del país, en la medida que es la región que presenta mayor número de grupos armados irregulares en disputa de los territorios (Unidad de Víctimas, 2012). La posibilidad de generar conexiones marítimas rápidas con Centro América, las particularidades y complejidades de su topografía, la cantidad de hectáreas sembradas de coca y la presencia histórica de organizaciones armadas ilegales, hicieron de esta región una de las más violentas, y por ende, más generadora de víctimas en Colombia.

La región Pacífica ha sido espacio de confrontación de los distintos grupos armados, especialmente aquellas subregiones en donde hay presencia de cultivos ilícitos, o en aquellas en las que hay corredores de movilidad estratégicos para conectar el centro con el sur del país, o las zonas fronterizas. Para el año 2002, la presencia de las FARC en la región era muy fuerte, especialmente en el norte del departamento del Chocó (zona de frontera con Panamá) y los departamentos del 
Valle Cauca y el Cauca. En el primer caso, buscando el control de las zonas de siembra de coca y marihuana; y en el segundo por la salida fronteriza al Ecuador.

Para el año 2015, el panorama se ha complejizado y agravado, ya que las FARC siguen ocupando los mismos territorios, pero han brotado de forma paulatina grupos armados ilegales asociados con el narcotráfico y con formas de autodefensa ilegal, lo que en definitiva ha traído confrontaciones y hostilidades que han afectado a la población civil, principalmente. El norte del departamento del Chocó se transformó radicalmente, las FARC salieron definitivamente de la zona, y en su lugar emergieron grupos ilegales vinculados al narcotráfico y a la minería ilegal.

\section{- Región Orinoquia}

En el caso de la región de la Orinoquia, en lo que respecta a pobreza se encuentra claramente divida en dos dinámicas territoriales. La subregión que cubre Arauca $(35,91 \%)$, Casanare $(35,55 \%)$ y Meta $(25,03 \%)$ se encuentra entre los niveles departamentales nacionales de presencia media-alta de pobreza. Por el otro lado, departamentos como Vichada (66,95\%) se encuentran en los niveles más altos de presencia de NBI a nivel nacional. Esta región concentra una población de comunidades indígenas entre el norte, centro y oriente, en el sur y occidente del departamento no presenta mayor presencia. En el norte, departamentos de Casanare y Arauca, se pueden encontrar comunidades como los U'wa, Hitnu, Betoye, Chiricoa, Tsiripu, Eduria, Wipijiwi, Yaruro, Misiguare. Ya en el oriente y sur oriente, departamentos de Vichada y Meta, están ubicadas comunidades como los Saliba, Piacopo, Kuiba, Amoruá, Guayabero y Achagua.

Estos pueblos indígenas se encuentran en 96 resguardos (DNP, 2014), en donde se halla una sobreposición de elementos como: pueblos incluidos en el Auto 004 de 2009, pueblos en vía de extinción y al mismo tiempo, tres de los pueblos nómadas que habitan en Colombia, los Guayaberos, Hitnus y Sikuanis, que por sus características culturales, se desplazan a lo largo de un territorio definido según los cambios estacionales y agrícolas. Los pueblos indígenas que componen esta región, se han visto influenciados y determinados por las dinámicas que han traído consigo la extracción de recursos naturales y el conflicto armado en el país. La extracción de petróleo y la concentración en el sector agrícola y pecuario con el cultivo de la palma de aceite, yuca, maíz, plátano, tabaco, entre otros; ha transformado la economía y las dinámicas de intercambio en las comunidades indígenas de la región.

Partiendo de lo anterior, la Orinoquia colombiana ha sido el escenario de los momentos de mayor tensión en la historia del país, por cuenta del auge que tuvo el Bloque Oriental de las FARC en la década de los años 90, y por la proliferación de estructuras armadas vinculadas a las AUC. De allí que en zonas específicas reporte altos índices de hechos victimizantes, huellas claras del paso del conflicto 
armado interno por esa zona del país. La situación en la subregión cercana a la ciudad de Villavicencio es completamente distinta, reportando unos indicadores cercanos a los que se presentan en la región Andina, y con zonas que incluso no reportan víctimas reconocidas.

\section{- Región Amazónica}

Finalmente, la Región Amazónica ofrece un panorama heterogéneo frente a la forma de reparto de pobreza en el territorio, a nivel departamental. Se encuentra en el primer grupo, departamentos como Putumayo (36,01\%) y Guaviare (39,89\%), con mejores niveles en cuanto a NBI. Por el otro lado, se en un segundo grupo está Caquetá (41,72\%), Amazonas (44,41\%) y Vaupés (54,77\%), con niveles un poco inferiores y ubicándose en un índice medio-alto de NBI. Finalmente se encuentra Guainía $(60,62 \%)$ la cual se localiza entre los departamentos a nivel nacional con mayor presencia de pobreza.

La Región Amazonia es la que mayor cantidad de pueblos indígenas integra y al mismo tiempo, la que mayor extensión de tierra tiene en resguardos indígenas, sumando en total 190 resguardos (DNP, 2014). El 17\% de la población amazónica (CEPAL, 2013) corresponde a los más de 50 diferentes pueblos. Al igual que en la región de Orinoquia, la Amazonia colombiana es el escenario en el que habitan otras dos poblaciones nómadas del país, los Ingas, y los Nukak Makú. Al igual que los otros tres, éstos hacen parte de la lista de los 34 pueblos incluidos en el Auto 004, ya que ambos se han visto seriamente afectados por el conflicto armado y las intervenciones empresariales que han interrumpido sus dinámicas ancestrales y culturales.

Distintas comunidades hacen presencia a lo largo y ancho del territorio amazónico. En la parte nororiental, departamentos de Vaupés y Guainía, hay comunidades como los Nukak Makú, Puinave, Curripaco, Carapana, Cubeo, Yurutí, Taiwano, Pisamira, Carapana, Tatuyo, Piratapuyo, Jupda, Bara y Tuyaca. En la parte Occidental, departamentos de Caquetá y Putumayo, comunidades como los Inga, Kofan, Coreguaje, Kichwa, Siona y Kamentza. Y finalmente, en el sur, departamento de Amazonas, se encuentran la amplia cantidad de comunidades como los Miraña, Andoque, Huitoto, Nonuva, Muinane, Carijona, Ocaina, Yauna, Letuama, Yucuma, Matapí, Tanimuca, Barasana, Tariano, Desana, Macuna, Kawiyavi, Yuri y Tucano.

La región podría prestarse para lecturas territorialmente incorrectas, ya que hay amplias zonas con bajos niveles de víctimas, pero que a su vez son zonas con unos niveles de poblamiento muy bajos, e incluso inexistentes (especialmente en zonas selváticas), lo que significa que la lectura de esta región debe hacerse a partir de esta especificidad. La Orinoquia y la Amazonia colombiana, reportan 
unas situaciones muy similares en lo que tiene que ver con el conflicto armado colombiano; las dos regiones están determinadas por la historia del Frente Oriental de las FARC, y la estrategia desarrollada por esta organización para controlar los entramados territoriales que conectan a Colombia con Brasil, Perú y Venezuela (Fundación Ideas para la Paz, 2012).

Esta zona fronteriza ha sido el escenario de unas dinámicas de criminalidad transnacional, debido a la configuración de corredores estratégicos por lo que entraban armas de fuego, y se comercializaban narcóticos (década de los años 90) e insumos y contrabando de minería ilegal (en la actualidad). Así mismo, en esta zona, especialmente en la Orinoquia, se armaron grupos de autodefensas ilegales en las regiones en las que existían yacimientos petrolíferos, o en las zonas ganaderas (Fuerza Aérea Colombiana, 2016).

\section{"Satena es la punta de lanza de todas esas regiones"}

Teniendo en cuenta todos los aspectos mencionados en las tres regiones señaladas, el rol que desempeña Satena es fundamental no solo por brindarle apoyo y presencia a las poblaciones étnicas y económicamente más necesitadas con labores como transportar medicinas y alimentos a estas regiones, sino por inspirar la gestión de la mejora de la infraestructura aeroportuaria para que puedan llegar aviones más grandes y se puedan realizar circuitos de rutas a varias horas del día para aumentar su cobertura. El Mayor General Lozano asegura que la ayuda del gobierno y la Aerocivil y los convenios a los que se llega con las alcaldías y gobernaciones, ha sido fundamental para este proceso de mejora, ya que se entiende que a medida que la infraestructura sea mejor, pueden tener más opciones de ingresos económicos que ayuden a mejorar la situación económica y social de cada uno de los destinos a los que llega Satena.

De igual manera, el presidente de la aerolínea asegura que entre más personas, tanto turistas como habitantes de los diferentes destinos, viajen por Satena, mayor va a ser el interés de las grandes aerolíneas comerciales en cubrir los diferentes municipios y pequeñas ciudades y así incrementar y fomentar el desarrollo interno de estas regiones. Frente a esto, el Mayor General Lozano explica que Satena fue la primera aerolínea en llegar a Yopal, Casanare cuando "aún era un potrero y tocaba ahuyentar a las vacas para poder aterrizar" y que fue gracias al crecimiento de los pasajeros que entraban y salían del municipio que Avianca y otras grandes aerolíneas entraron en ese mercado para competir con ellos, acto que generó que Satena se hiciera a un lado no sin antes sentirse orgullosos, que fue gracias a ellos, que los habitantes de esta ciudad pudieran tener el contacto directo con otras ciudades capitales.

Sumado a lo anterior, y como parte esencial del trabajo social al que responde Satena, hay un gran porcentaje de la población ya descrita que habita en estas 
regiones, que ha hecho uso de esta aerolínea como una solución a emergencias médicas como graves accidentes o incluso embarazos críticos. Sin embargo, a esta población también la ha tenido que asistir directamente la Fuerza Aérea Colombiana debido a que los pobladores consideran que el precio de los pasajes es muy alto y no pueden acceder a los servicios de la aerolínea.

El Mayor General Lozano explica que ese tipo de operaciones en donde los helicópteros de la FAC entran a hacer ese servicio social, sale sumamente costoso y que por lo tanto es Satena la que debe cerciorarse de cumplir también ese tipo de acción social. Para eso, la aerolínea está interesada en realizar convenios con las alcaldías y gobernaciones para crear subsidios a las personas que por motivos críticos de salud necesiten salir de sus regiones y poder llevarlas a un costo mucho más accesible a las principales ciudades del país. Actualmente la única población que cuenta con dicho subsidio son los habitantes y oriundos de San Andrés y Providencia para que puedan realizar el vuelo entre las dos islas a mitad del precio que se vende para el turismo en general.

"Servir, planear y desarrollar" son los tres aspectos que señala el Mayor General Lozano como bases del servició social que cumple Satena para el desarrollo del país y asegura que se está trabajando para que dentro poco tiempo se pueda incrementar la cantidad de destinos y seguir apoyando a la población que se encuentra más alejada del centro del país con acciones fundamentales como el hecho de no haber subido los precios de los pasajes aún incluso con la fuerte subida del dólar en el año 2015. Esto con el fin de que la población que más necesita llegar a estos destinos pueda hacer uso de la infraestructura aérea de Satena.

\section{Satena y su papel en el posconflicto}

Durante el conflicto armado en el país, Satena se ha encargado de continuar su labor social de acercar a las personas a los lugares que se han visto afectados por la violencia. Las regiones afectadas se beneficiaban con el arribo de la aerolínea durante el conflicto por la afluencia de medicamentos y comida para toda la población. Ahora, a puerto de entrar en un escenario de posconflicto se planea que se pueda incrementar el número de vuelos y de destinos para que el turismo pueda tener más acogida, como lo anhela el Mayor General Lozano quien se ha encargado de recorrer cada uno de los lugares a los que llega Satena para deleitarse del paisaje que pocos han tenido acceso, hasta ahora.

"Hay una función social de dar a conocer el país a todas las personas que no lo han podido hacer por causas del orden público" agrega el Mayor General de la Fuerza Aérea Colombiana. Uno de los ejemplos de esto es Villa Garzón en el Putumayo, una de las zonas más afectadas por el conflicto armado en el país, es ahora un lugar que se está promocionando como un destino turístico y que por medio de la publicidad de Satena se busca que se conozca a profundidad el país. 
En el marco de estos escenarios de posconflicto, y adicionalmente a los conflictos sociales que puedan emerger en cuanto a la sobreposición de actividades extractivas y áreas de reserva ambiental, estos territorios periféricos sufren dos tipos de amenazas. Por un lado, la presencia de economías ilícitas como la explotación maderera y minera ilegal, que constituyen dos graves amenazas en cuanto a la preservación ambiental. El avance de la minería ilegal de coltán y tungsteno en PNN como el Puinawai y Amacayacu es una amenaza considerable que deber ser enfrentada contundentemente.

Por el otro lado, y en clave de posconflicto, una posible migración de la producción de coca de las zonas históricas (Macarena, Catatumbo, Putumayo, entre otras) -por cuenta de los incentivos que puedan ofrecerse para los cultivadores tradicionales en el marco del posconflicto- a zonas como la fronteras amazónica donde por cuenta de características como zonas de difícil acceso, presencia de parques naturales y acceso a la frontera facilitarían el cultivo y transporte de narcóticos.

En un escenario de posconflicto, los desafíos se darán en cuanto a la llegada de nuevos actores con capacidad de copar los espacios que dejen las FARC en los territorios. Los riesgos de fragmentación en la desmovilización de las FARC es un escenario posible (McDermott, 2013), ante el cual también hay que contar con la criminalización de elementos de las FARC que continúen en el territorio delinquiendo. Este elemento de fragmentación y la transformación de la amenaza más allá del proceso de desmovilización tienen antecedentes en procesos como los del Ejército Popular de Liberación (EPL), las Autodefensas de Colombia (AUC).

Así mismo, se ha señalado el riesgo de la mimetización total de las estructuras armadas de las FARC con el narcotráfico, invisibilizando el accionar de sus hombres detrás de las fachadas construidas por los cárteles de la droga, y evadiendo las lecturas de macrocriminalidad que hoy se efectúan. Esto implicará una labor de inteligencia policial y militar muy fuerte, que supere fenómenos como el que vivió el San Salvador luego de la desmovilización del FMNL, en donde esta organización siguió operando a través de pequeños grupos criminales (BENíteZ, 1993, p. 8). Este fenómeno de invisibilización es muy similar al que se produjo con la desmovilización de las AUC en el año 2005, con una aparición de innumerables bandas criminales, de las se desconocía su estructura y zonas de operación (ACNUR, 2006).

Ante esta situación, Rettberg (2002) ha señalado que se debe generar una transformación completa del concepto de seguridad, trasladándolo de la seguridad nacional a la seguridad ciudadana; este cambio no es un problema meramente semántico o conceptual, ya que reestructura los roles de las Fuerzas Militares, y pone en una situación protagónica a la Policía Nacional. Por ello, se ha tornado fundamental reflexionar sobre el futuro de una Fuerza Militar en un escenario de posconflicto, señalando que su legitimidad y continuidad va más allá del conflicto mismo. 


\section{CONSIDERACIONES FINALES}

En el contexto específico de los municipios a los que tiene como destino Satena, el reto de la capacidad de llevar desarrollo y bienestar a las comunidades se ve incrementado por obstáculos como la geografía y la dispersión. Con unas comunidades viviendo en 15 de los 16 municipios/corregimientos departamentales dentro de la categoría de "rural remoto" (DNP y Misión Rural, 2015), todo tipo de inversión se incrementa en tiempo y recursos, agravado por fenómenos de corrupción. Sin embargo, este reto social de Satena sigue siendo vital ya que el bienestar de las comunidades redundara en la posibilidad de cerrarle espacio a grupos armados y economías ilegales, construyendo legitimidad en el Estado y sus instituciones en el territorio. El cómo se puede avanzar en este tipo de desafíos y el rol que distintos actores (Sector Público, Sector Privado, Fuerzas Armadas, Academia, entre otros) puedan tener en este proyecto de llevar bienestar a estas zonas, constituye un reto al trabajo conjunto y la innovación de Satena.

Desde otro punto de vista, el desarrollo de los lugares acá mencionados no puede depender únicamente de la aerolínea que con su esfuerzo ha tratado de llegar cada vez a más lugares del país. Hay una serie de retos institucionales, los cuales se manifiestan por las necesidades de fortalecer todo el entramado de instituciones estatales que constituyen la presencia del Estado en el territorio. En el entramado de retos sociales y ambientales que surgen en estos territorios de frontera, la necesidad de una respuesta completa y coherente por parte del Estado constituye una necesidad. En este caso, los retos institucionales pasan desde la atención del Gobierno central, el fortalecimiento de los distintos actores gubernamentales regionales y locales, y el combate frontal contra la corrupción. En cuanto a la atención por parte del gobierno central, la capacidad de destinar recursos para este territorio continúa siendo prioritario por cuenta de la crítica situación en que se encuentra solo viendo indicadores como NBI en los municipios/corregimientos.

La necesidad del fortalecimiento de las entidades gubernamentales a nivel regional y local. En este caso se requiere mejorar las capacidades y recursos con que cuentan funcionarios e instituciones para el cumplimiento de su misión. En un ejemplo, como el combate frontal contra la minería ilegal, en el escenario actual, la asimetría entre los recursos y capacidades de organizaciones criminales hacen imposible que el Estado pueda estar vigilante sobre el daño a los ecosistemas. Se requiere de una gran articulación entre autoridades locales, organismos de control, autoridades ambientales (Corporaciones Autónomas Regionales) y Fuerza Pública, en donde las autoridades a nivel local y regional tengan la capacidad de estar a la cabeza de estos hechos.

Por último, el reto institucional se refiere a la corrupción como uno de las amenazas más grandes en cuanto a la capacidad de fortalecer institucionalmente 
al Estado en los territorios, pero también de fortalecer la misma legitimidad para toda la intervención del mismo. Respecto a la corrupción como un obstáculo en el fortalecimiento institucional, es claro que las innumerables necesidades de estos territorios no van a encontrar solución si la destinación de los recursos nunca llega a las comunidades sino en el enriquecimiento de unos pocos funcionarios y particulares. En el escenario de posconflicto, y en el marco de la inversión territorial que conlleva, se corre el mismo riesgo.

\section{REFERENCIAS}

ACNUR (2005) Algunos indicadores sobre la situación de los Derechos Humanos en Antioquia. Bogotá, ACNUR y Observatorio del programa presidencial para los Derechos Humanos y el DIH.

ACNUR (2006) Diagnóstico del Departamento del Meta. Colombia, Naciones Unidas-ACNUR.

Benítez, R (1993) Condiciones de seguridad en San Salvador tras los acuerdos de paz. San Salvador, Nueva Sociedad.

DANE (2016) Necesidades Básicas Insatisfechas. Tomado de: https://www.dane. gov.co/index.php/estadisticas-por-tema/pobreza-y-condiciones-de-vida/ necesidades-basicas-insatisfechas-nbi

DNP, Departamento Nacional de Planeación. (2014). Aplicativo de Consulta de Resguardos. Disponible en la página web: http://sige.dane.gov.co:81/ resguardos/map.phtml

Fuerza Aérea Colombiana (2016). La Fuerza Aérea Colombiana y el cese del conflicto armado: Victorias desde el aire. Bogotá, Fuerza Aérea Colombiana.

McDermont, J. (2013). "Posibles escenarios de una fragmentación o criminalización de las FARC”. Insight Crime. Centro de Investigación de Crimen Organizado.

Unidad de Víctimas. (2012). Informe nacional de desplazamiento forzado en Colombia 1985 a 2012. Colombia, Unidad de Víctimas. 